PROCEEDINGS OF THE

AMERICAN MATHEMATICAL SOCIETY

Volume 33, Number 2, June 1972

\title{
COALGEBRAS, SHEAVES, AND COHOMOLOGY
}

\author{
D. H. VAN OSDOL ${ }^{1}$
}

\begin{abstract}
The category of sheaves on the topological space $X$ with values in the algebraic category $\mathscr{A}$ is shown to be cotripleable under the stalk category $\mathscr{A}^{|x|}$. If $K$ is a field then the category of $K$-coalgebras is shown to be cotripleable under the category of $K$-vector spaces. This makes possible the interpretation of the first group of the associated triple cohomology complex. In particular, for coalgebras our $H^{1}$ is isomorphic to Jonah's $H^{2}$.
\end{abstract}

1. Introduction. The theory of triples $T$ and their associated $T$-algebras has been a unifying force in mathematics as well as a rapidly expanding area of research ([1], [3], [6], [8], [9]). The dual theory of cotriples $G$ and their associated $G$-coalgebras has been relatively neglected (however, see the article of Applegate and Tierney in [3]). We suggest that this neglect is unwarranted, and prove in this note that two important categories are categories of (cotriple-theoretic) coalgebras.

First, the stalk functor from algebraic sheaves on the topological space $X$ to sheaves on the space $X$ made discrete is cotripleable. Second, if $K$ is a field then the forgetful functor from $K$-coalgebras to $K$-vector spaces is cotripleable. We enunciate the dual of Beck's classification theorem [1] and use it to give an interpretation of the cohomology for sheaves and coalgebras.

Throughout this paper, if $A$ and $B$ are objects of a category $\mathscr{A}$ then $(A, B)$ will denote the set of $\mathscr{A}$-morphisms from $A$ to $B$; whereas if $\mathscr{A}$ is a small category and $\mathscr{B}$ is a category then $\mathscr{B}^{\mathscr{A}}$ will denote the category whose objects are covariant functors $\mathscr{A} \rightarrow \mathscr{B}$ and whose morphisms are natural transformations of functors. A symbol $X$ will denote either the object $X$ or the identity morphism on $X$.

2. Sheaves of algebras. Let $\mathscr{A}$ be an algebraic category [9]. Among the algebraic categories are all the usual categories of algebras, for example

Presented to the Society, September 3, 1971; received by the editors September 27, 1971.

AMS 1970 subject classifications. Primary 18C15, 18F20, 18G10, 18H40; Secondary 55B30, $18 \mathrm{D} 35$.

Key words and phrases. Sheaves of algebras, cotripleable, coalgebras, principal objects, cogroup object, extension, Jonah cohomology.

1 Work supported by National Science Foundation grant GP-29067.

c. American Mathematical Society 1972 
the categories of groups, $R$-modules, rings, Lie rings, algebras, and Lie algebras. Let $X$ be a topological space and let $\mathscr{P}(X, \mathscr{A})$ (respectively $\mathscr{F}(X, \mathscr{A})$ ) be the category of presheaves (respectively sheaves) on $X$ with values in $\mathscr{A}$ [7]. Let $|X|$ be the discrete category on the set $X$.

The stalk functor $S: \mathscr{P}(X, \mathscr{A}) \rightarrow \mathscr{A}^{\mid X_{\mid}}$is defined by $(S P)\{x\}=P_{x}=$ colim $P V$ where the colimit is taken over all open subsets $V$ of $X$ which contain $x$; note that this is a directed colimit. The right adjoint to $S$, $Q: \mathscr{A}^{|x|} \rightarrow \mathscr{P}(X, \mathscr{A})$, is defined as follows: given $\left\{A_{x}\right\}$ in $\mathscr{A}^{|x|}$ and an open subset $V$ of $X,\left(Q\left\{A_{x}\right\}\right) V=\prod A_{x}$ where the product is taken over all points $x$ in $V$. The definitions of $S$ and $Q$ on morphisms are obvious. Actually since $Q\left\{A_{x}\right\}$ is a sheaf [7], we have the "same" adjoint pair $\mathscr{F}(X, \mathscr{A}) \rightarrow \mathscr{A}^{|X|} \rightarrow \mathscr{F}(X, \mathscr{A})$. Note that $Q S$ is the Godement standard construction [4].

THEOREM 1. $S: \mathscr{F}(X, \mathscr{A}) \rightarrow \mathscr{A}^{\left|{ }^{\mid}\right|}$is crudely cotripleable, and $\mathscr{F}(X, \mathscr{A})$ is cocomplete.

Proof. Since $\mathscr{A}$ is complete, so is $\mathscr{F}(X, \mathscr{A})$. For sheaves in an algebraic category, a morphism of sheaves is an isomorphism if and only if it is an isomorphism on each stalk [4]. In an algebraic category directed colimits commute with finite limits [9]. Hence we have verified the conditions of the CCT [1].

Thus there exists an equivalence $\Psi^{\prime \prime}:\left(\mathscr{A}^{|X|}\right)_{G \rightarrow \mathscr{F}}(X, \mathscr{A})$. Its composition with the comparison functor $\Psi: \mathscr{P}(X, \mathscr{A}) \rightarrow\left(\mathscr{A}^{|X|}\right)_{G}$ is easily described: $\Psi^{\prime \prime} \Psi=$ equalizer $(\eta Q S, Q S \eta)$. One can then use Theorem 1 to prove the following:

COROLLARY 2. $\Psi^{\prime \prime} \Psi$ is the associated sheaf functor, that is, $\Psi^{\prime \prime} \Psi \dashv i$ where $i: \mathscr{F}(X, \mathscr{A}) \rightarrow \mathscr{P}(X, \mathscr{A})$ is the inclusion functor.

For interpretation of these results, see [11].

3. Coalgebras. Let $K$ be a field and let $\mathscr{H}$ be the category of $K$ modules (vector spaces). A $K$-coalgebra is a three-tuple $(C, \Delta, c)$ where $C$ is a $K$-module and $\Delta: C \rightarrow C \bigcirc C, c: C \rightarrow K$ are $K$-module homomorphisms such that $(c @ C) \cdot \Delta=C=(C @ c) \cdot \Delta$ and $(\Delta \subseteq C) \cdot \Delta=(C \Delta) \cdot \Delta$. A morphism of $K$-coalgebras, $f:(C, \Delta, c) \rightarrow\left(C^{\prime}, \Delta^{\prime}, c^{\prime}\right)$, is a $K$-module homomorphism $f: C \rightarrow C^{\prime}$ such that $\Delta^{\prime} \cdot f=(f \times f) \cdot \Delta$ and $c^{\prime} \cdot f=c$. Let $\mathscr{C}$ be the category of $K$-coalgebras and $S: \mathscr{C} \rightarrow \mathscr{H}$ the obvious forgetful functor.

TheOREM 3. $S: \mathscr{C} \rightarrow \mathscr{H}$ is cotripleable.

Proof. It is proved in [10] that $S$ has a right adjoint. Clearly $S$ reflects isomorphisms. Given morphisms

$$
C_{1} \rightrightarrows C_{2}
$$


such that

$$
E \stackrel{i}{\rightarrow} S C_{1} \rightrightarrows S C_{2}
$$

is an absolute equalizer, we give $E$ a coalgebra structure in the following way. Since $F(M)=M \otimes M, F(f)=f \otimes f$ defines a functor $\mathscr{F}: \mathscr{M} \rightarrow \mathscr{M}$, it follows that

$$
E \otimes E \rightarrow S C_{1} \otimes S C_{1} \rightrightarrows S C_{2} \otimes S C_{2}
$$

is an equalizer. Hence there is a unique $\Delta: E \rightarrow E \otimes E$ such that $(i \otimes i) \cdot \Delta=$ $\Delta_{1} \cdot i$. If we let $e=c_{1} \cdot i$, then it is easy to verify that $(E, \Delta, e)$ is a coalgebra and

$$
E \rightarrow C_{1} \rightrightarrows C_{2}
$$

is the equalizer in $\mathscr{C}$. Hence we have verified the conditions of the PCT [8].

4. Beck's classification theorem. Let $S: \mathscr{A} \rightarrow \mathscr{B}$ be cotripleable with right adjoint $Q: \mathscr{B} \rightarrow \mathscr{A}$. For each object $A$ of $\mathscr{A}$ we get a semisimplicial complex $T^{*} A$, where in dimension $n \geqq 0$ we have $(Q S)^{n+1} A$ and for each $i \leqq n+1, \quad \eta_{i}=(Q S)^{i} \eta(Q S)^{n+1-i} A:(Q S)^{n+1} A \rightarrow(Q S)^{n+2} A$. Suppose $\mathscr{B}$ is finitely cocomplete (which implies that $\mathscr{A}$ is finitely cocomplete) and let $C=(C, \gamma: C \rightarrow C \amalg C, c: C \rightarrow 0, l: C \rightarrow C)$ be an abelian cogroup object in $\mathscr{A}$. Then $\left(C, T^{*} A\right)$ is a cochain complex, where $d:\left(C, T^{n+1} A\right) \rightarrow\left(C, T^{n+2} A\right)$ is defined by $d(f)=\sum_{i=0}^{n+1}(-1)^{i} \eta_{i} \cdot f$. We define $H^{n}(C, A)=$ the $n$th homology group of $\left(C, T^{*} A\right)$.

Theorem 4. $H^{0}(C, A) \cong(C, A)$.

Proof. See Beck [1].

To interpret $H^{1}(C, A)$ we need some more definitions. A pair $(D, \rho)$ is a $C$-object if $D$ is an object of $\mathscr{A}, \rho: D \rightarrow D \amalg C$ in $\mathscr{A},(D \amalg \gamma) \cdot \rho=$ $(\rho \amalg C) \cdot \rho$, and $(D \amalg c) \cdot \rho=D$. A $C$-principal object under $A$ is a morphism $i: A \rightarrow D$ in $\mathscr{A}$ where $(D, \rho)$ is a $C$-object and:

(i) $(i \amalg C) \cdot i_{1}=\rho \cdot i$ where $i_{1}: A \rightarrow A \amalg C$ is the injection.

(ii) For each pair $f_{1}, f_{2}: D \rightarrow X$ in $\mathscr{A}$ such that $f_{1} \cdot i=f_{2} \cdot i$ there exists a unique $g: C \rightarrow X$ in $\mathscr{A}$ such that $\left(f_{1} \amalg g\right) \cdot \rho=f_{2}$.

(iii) There is given as part of the structure a map $s: S D \rightarrow S A$ such that $s \cdot S i=S A$.

A morphism $(D, \rho) \rightarrow\left(D^{\prime}, \rho^{\prime}\right)$ of $C$-principal objects under $A$ is an $\mathscr{A}$-morphism $f: D \rightarrow D^{\prime}$ such that $f \cdot i=i^{\prime}$ and $(f \amalg C) \cdot \rho=\rho^{\prime} \cdot f$. Let $\mathscr{P O}$ be the category of $C$-principal objects under $A$ and their morphisms.

THEOREM 5. $H^{1}(C, A)$ is isomorphic to the set of connected components of $\mathscr{P O}$.

Proof. See Beck [1].

5. Specific examples. We now apply Theorem 5 to the situations studied in $\S \S 2$ and 3. 
EXAMPLE 1. If $\triangle$ is the category of abelian groups and homomorphisms then every object $C$ in $\mathscr{\mathscr { F }}(X, \mathscr{A})$ is an abelian cogroup where $\gamma: C \rightarrow C \amalg C$ is the diagonal map, $c: C \rightarrow 0$ is the zero map, and $l: C \rightarrow C$ is inversion. If $A$ is another sheaf of abelian groups then $H^{1}(C, A)$ will be in one-one correspondence with the set of equivalence classes of short exact sequences $O \rightarrow A \rightarrow D \rightarrow C \rightarrow O$ of sheaves of abelian groups such that the sequence of abelian groups $0 \rightarrow A_{x} \rightarrow D_{x} \rightarrow C_{x} \rightarrow 0$ is split (as abelian groups) for each $x$ in $X$. Of course two such sequences are equivalent if there exists a commutative diagram

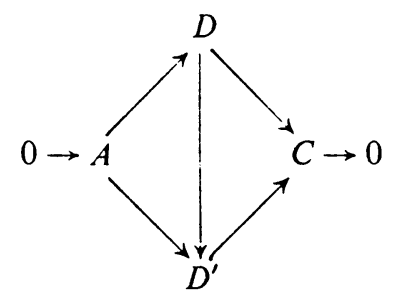

of sheaves of abelian groups. When we take $C=Z=$ the constant sheaf of integers, we conclude that $\left(Z, T^{*} A\right) \cong \Gamma T^{*} A$. Hence $H^{n}(Z, A) \cong H^{n}(X, A)$, the usual cohomology of $X$ with coefficients in $A$ (since $T^{*} A$ is the Godement resolution of $A$ ) [4]. On the other hand, the sequence $0 \rightarrow A_{x} \rightarrow$ $D_{x} \rightarrow Z_{x} \rightarrow 0$ will automatically split because $Z_{x}$ is free. Hence $H^{1}(X, A) \cong$ $H^{1}(Z, A) \cong \operatorname{Ext}^{1}(Z, A)$. Similarly, if $C$ has projective stalks then $H^{1}(C, A) \cong \operatorname{Ext}^{1}(C, A)$.

Example 2. One can define $H^{0}(C, A)$ and $H^{1}(C, A)$, essentially as above, when $C$ is a not-necessarily-abelian cogroup (see [1]). If $\mathscr{A}$ is the category of commutative $K$-algebras, then the cogroups in $\mathscr{A}$ are the affine algebraic groups [2]. Let $C$ be an object in $\mathscr{F}(X, \mathscr{A})$ which is a sheaf of affine algebraic groups: then $C$ is a cogroup object in $\mathscr{F}(X, \mathscr{A})$. Let $A$ be a sheaf of commutative $K$-algebras. Then $H^{1}(C, A)$ is in one-one correspondence with equivalence classes of sheaves of $K$-algebras $D$ such that $D$ contains $A$ as a subsheaf and $D_{x} \cong A_{x} \cong C_{x}$ for each $x$ in $X$. In other words, $H^{1}(C, A)$ tells how many inequivalent sheaves there are which have stalks $A_{x} C_{x}$ and contain $A$ as a subsheaf.

REMARK. Example 2 is typical for sheaves with values in some algebraic category $\mathscr{A}$. Roughly speaking, $H^{1}(C, A)$ will classify sheaves containing $A$ and having stalks $A_{x} \amalg C_{x}$.

EXAMPLE 3. Let $\mathscr{C}$ be the category of $K$-coalgebras defined in $\S 3$, and let $C$ be a coalgebra. The comma category $(C, \mathscr{C})$ has objects $C \stackrel{i}{\rightarrow} \Gamma$ in $C$ and maps $C_{\rightarrow}^{i} \Gamma_{\rightarrow}^{f} \Gamma^{\prime}=C \stackrel{i}{\rightarrow} \Gamma^{\prime}$. One can show that $(C, S):(C, \mathscr{C}) \rightarrow(S C, \mathscr{M})$ is cotripleable, where $(C, S)(C \rightarrow \Gamma)=S C \rightarrow S \Gamma$ and similarly for maps. The dual situation is considered by Beck in [1]. Let $\operatorname{Coab}(\mathrm{C}, \mathscr{C})$ be the 
category of abelian cogroups in $(C, \mathscr{C})$; we claim that $\operatorname{Coab}(C, \mathscr{C})$ is equivalent to the category of $C$ - $C$-comodules. Given an abelian cogroup $i: C \rightarrow \Gamma$, we first note that the cogroup counit $d: \Gamma \rightarrow C$ yields an isomorphism of $K$-modules $\Gamma \cong C \oplus M$ where $M$ is the cokernel of $i: C \rightarrow \Gamma$. Replacing $\Gamma$ by $C \oplus M, i: C \rightarrow C \oplus M$ becomes the injection into the first factor, $i(x)=(x, 0)$. Using the matrix notation of Jonah [5], the coalgebra comultiplication $\gamma: C \oplus M \rightarrow(C \ominus M) \otimes(C \oplus M)$ can be represented by

$$
\gamma=\left[\begin{array}{cc}
\Delta & h \\
0 & m_{l} \\
0 & m_{r} \\
0 & m
\end{array}\right]
$$

where $\Delta: C \rightarrow C \otimes C$ is the coalgebra costructure, $h: M \rightarrow C \otimes C, m_{l}: M \rightarrow$ $C \otimes M, m_{r}: M \rightarrow M \otimes C$, and $m: M \rightarrow M \otimes M$. Since $d=[C 0]$ is a map of coalgebras, $(d \otimes d) \cdot \gamma=\Delta \cdot d$ implies $h=0$. Also if $c: C \rightarrow K$ is the coalgebra counit and $c^{\prime}: C \oplus M \rightarrow K$ is the coalgebra counit then $d \cdot c=c^{\prime}$ implies $c^{\prime}=\left[\begin{array}{c}c \\ 0\end{array}\right]$.

We are next interested in the cogroup comultiplication. Notice that the coproduct of $i: C \rightarrow C \oplus M$ with itself is $C \rightarrow C \oplus M \oplus M$, and hence the cogroup comultiplication will be a coalgebra map $e: C \oplus M \rightarrow C \oplus M \oplus M$. One checks that the coalgebra costructure $\delta: C \oplus M \oplus M \rightarrow(C \oplus M \oplus M) \otimes$ $(C \oplus M \oplus M)$ is represented by

$$
\delta=\left[\begin{array}{ccc}
\Delta & 0 & 0 \\
0 & m_{l} & 0 \\
0 & 0 & m_{l} \\
0 & m_{r} & 0 \\
0 & m & 0 \\
0 & 0 & 0 \\
0 & 0 & m_{r} \\
0 & 0 & 0 \\
0 & 0 & m
\end{array}\right] .
$$

Since

$$
e=\left[\begin{array}{cc}
C & 0 \\
0 & M \\
0 & M
\end{array}\right]
$$


is a coalgebra map, $(e \otimes e) \cdot \gamma=\delta \cdot e$ implies that $m=0$. Hence

$$
\gamma=\left[\begin{array}{cc}
\Delta & 0 \\
0 & m_{l} \\
0 & m_{r} \\
0 & 0
\end{array}\right] .
$$

Now $[(C \oplus M) \otimes \gamma] \cdot \gamma=[\gamma \odot(C \oplus M)] \cdot \gamma$ says that $(\Delta \otimes M) \cdot m_{l}=\left(C \odot m_{l}\right) \cdot m_{l}$, $(M \otimes \Delta) \cdot m_{r}=\left(m_{r} \otimes C\right) \cdot m_{r}, \quad$ and $\left(C \subseteq m_{r}\right) \cdot m_{l}=\left(m_{l} \otimes C\right) \cdot m_{r}$. Moreover, $\left[c^{\prime} \otimes(C \oplus M)\right] \cdot \gamma=C \ominus M$ implies $(c \subseteq M) \cdot m_{l}=M$ and $\left[(C \ominus M) \Omega c^{\prime}\right] \cdot \gamma=$ $C \oplus M$ implies $(M \otimes c) \cdot m_{r}=M$. Such a $K$-module $\left(M, m_{l}, m_{r}\right)$ is precisely a two-sided $C$-comodule, and hence $\operatorname{Coker:} \operatorname{Coab}(C, \mathscr{C}) \rightarrow C$ - $C$-comodules defines a functor.

On the other hand, given a two-sided $C$-comodule $\left(M, m_{l}, m_{r}\right)$ we define $\Gamma=C \oplus M$ as a $K$-module. Let

$$
\gamma=\left[\begin{array}{cc}
\Delta & 0 \\
0 & m_{l} \\
0 & m_{r} \\
0 & 0
\end{array}\right]
$$

and $c^{\prime}=\left[\begin{array}{ll}c & 0\end{array}\right]$, thus making $\Gamma$ into a coalgebra and $i=\left[\begin{array}{l}C \\ 0\end{array}\right]$ into a coalgebra map. Finally

$$
e=\left[\begin{array}{cc}
C & 0 \\
0 & M \\
0 & M
\end{array}\right], \quad d=\left[\begin{array}{ll}
C & 0
\end{array}\right], \quad l=\left[\begin{array}{cc}
C & 0 \\
0 & -M
\end{array}\right]
$$

give $i: C \rightarrow \Gamma$ an abelian cogroup structure, and one verifies that this assignment is the functorial inverse to Coker. Using this category equivalence it is possible to reinterpret the group of morphisms $(i: C \rightarrow \Gamma$, $\left.i^{\prime}: C \rightarrow \Gamma^{\prime}\right)$ where $i$ is an abelian cogroup in $(C, \mathscr{C})$. Given a $C$ - $C$-comodule $M$ and coalgebra $\left(\Gamma^{\prime}, \gamma^{\prime}, c^{\prime}\right)$, a $K$-module morphism $f: M \rightarrow \Gamma^{\prime}$ is an $i^{\prime}$ coderivation from $M$ to $\Gamma^{\prime}$ if $\gamma^{\prime} \cdot f=\left(i^{\prime} \otimes f\right) \cdot m_{l}+\left(f \otimes i^{\prime}\right) \cdot m_{r}$ and $c^{\prime} \cdot f=0$. Then $\left(i, i^{\prime}\right)$ is in one-one correspondence with the group of all $i^{\prime}$ coderivations from $M$ to $\Gamma^{\prime}$, where $M=$ Coker $i$.

Applying Theorem 5 to this situation, we let $i: C \rightarrow \Gamma$ be an abelian cogroup in $(C, \mathscr{C})$ and interpret $H^{1}(i, C)$ where $C$ is the initial object in $(C, \mathscr{C})$. An $i$-principal object under $C$ will be a coalgebra map $j: C \rightarrow \Gamma^{\prime}$ such that $S j$ is isomorphic to the coproduct of $S i$ and $S C$, which of course is $S i$. Hence as $K$-modules, $\Gamma^{\prime}$ and $\Gamma=C \oplus M$ are isomorphic. It follows that if we let $J H^{2}(M, C)$ be Jonah's second cohomology group [5] then $H^{1}(i, C) \cong J H^{2}(M, C)$ where $M=$ Coker $i$. Note finally that one can compute $H^{n}(i, C)$ as the $n$th homology group of the complex $\operatorname{Coder}\left(M, T^{*} C\right)$. 


\section{REFERENCES}

1. J. M. Beck, Triples, algebras, and cohomology, Dissertation, Columbia University, New York, 1967.

2. A. Borel, Linear algebraic groups, Benjamin, New York, 1969. MR 40 \#4273.

3. B. Eckmann, Seminar on triples and categorical homology theory (ETH 1966/67), Lecture Notes in Math., no. 80, Springer-Verlag, Berlin and New York, 1969. MR 39 \#1511.

4. R. Godement, Topologie algébrique et théorie des faisceaux, Actualités Sci. Indust., no. 1252, Publ. Math. Univ. Strasbourg, no. 13, Hermann, Paris, 1958. MR 21 \#1583.

5. D. W. Jonah, Cohomology of coalgebras, Mem. Amer. Math. Soc. No. 82 (1968). MR 37 \#5267.

6. E. G. Manes, A triple miscellany: Some aspects of the theory of algebras over a triple, Dissertation, Wesleyan University, Middletown, Conn., 1967.

7. B. Mitchell, Theory of Categories, Pure and Appl. Math., vol. 17, Academic Press, New York, 1965. MR 34 \#2647.

8. R. Paré, Absolute coequalizers, Category Theory, Homology Theory and their Applications (Battelle Institute Conference, Seattle, Wash., 1968), vol. 1, Springer, Berlin, 1969, pp. 132-145. MR 39 \#5658.

9. B. Pareigis, Categories and functors, Academic Press, New York, 1970.

10. M. E. Sweedler, Hopf algebras, Math. Lecture Note Series, Benjamin, New York, 1969. MR 40 \#5705.

11. D. H. Van Osdol, Sheaves in regular categories, Exact Categories and Categories of Sheaves, Lecture Notes in Math., no. 236, Springer-Verlag, Berlin and New York, 1971, pp. 223-239.

Department of Mathematics, University of New Hampshire, Durham, New HAMPSHIRE 03824 